\title{
Intrauterine fetal death: Five years' experience in a secondary care hospital.
}

1. MBBS, FCPS

Senior Instructor Obs \& Gyn The Agha Khan University, Karachi, Pakistan

2. MBBS, MCPS, FCPS Associate Professor Obs \& Gyn HITEC IMS Taxila Cantt.

3. MBBS, FCPS

Instructor Obs \& Gyn Aga Khan University Karachi Pakistan.

4. MBBS, FCPS

Senior Instructor Research

Aga Khan University Karachi Pakistan.

5. MBBS, FCPS

Senior Instructor Research Associate

Aga Khan University Karachi Pakistan.

6. MBBS, FCPS

Associate Professor Obs \& Gyn Isra Medical College and Hospital Islamabad.

Correspondence Address:

Dr. Erum Saleem Khan Department of Obs Gyn

The Agha Khan University, Karachi, Pakistan.

erum959@hotmail.com

Article received on:

15/04/2020

Accepted for publication:

$30 / 11 / 2020$
Erum Saleem Khan', Mahwash Jamil', Reeta Chanderparkash ${ }^{3}$, Sheikh Irfan ${ }^{4}$, Sana Yousf ${ }^{5}$, Ayesha Basharat ${ }^{6}$

ABSTRACT... Objectives: To estimate the prevalence, preoperative, and operative risk factors associated with the higher risk of SSI in gynecologic cancer patients undergoing surgery in a tertiary care facility in a developing country. Study Design: Retrospective Study. Setting: Obstetrics and Gynecology Department, Agha Khan Hospital for Women Garden Karachi, Pakistan. Period: Jan 2012 to Dec 2016. Material \& Methods: Retrospective review of medical records of patients over a period of five years from Jan 2012 to Dec 2016 was done. Results: The study consisted of 56 cases of intrauterine fetal demise out of 4813 number of deliveries which occurred during the study duration. Overall estimated rate of intrauterine fetal demise was $11.6 \%$ (56/4813). Approximately $33.9 \%$ of cases did not have a clear cause of fetal death identified and hence were un-explained. In 56 cases, $28.6 \%$ had some form of congenital abnormality identified on ultrasound, 7.1\% $(n=4)$ had anemia, 23.2\% $(n=13)$ IUGR, 5.4\% $(n=3)$ placental abruption and $1.8 \%(n=1)$ of gestational diabetes and around $83.9 \%(n=47)$ had normal vaginal delivery and 8 were delivered by LSCS and only 1 patient had instrumental delivery. Fetal characteristics identified $50 \%(n=28)$ were males and $50 \%(n=28)$ were females. 4 fetuses were born with tight cord around their neck $58.1 \%$ were macerated stillbirths $(n=25)$ while $32.6 \%$ were fresh stillbirth. Conclusion: Antepartum intrauterine fetal death or stillbirths in the third trimester, affect the entire family. We conclude from our study that quality antenatal care with health education and timely interventions can reduce the number of intrauterine fetal deaths thus reducing the psychological sequelae.

Key words: $\quad$ Congenital Anomaly, Intrauterine Fetal Demise, Placental abruption, Stillbirth.

Article Citation: Khan ES, Jamil M, Chanderparkash R, Irfan S, Yousaf S, Basharat A. Intrauterine fetal death: Five years' experience in a secondary care hospital. Professional Med J 2021; 28(8):1076-1082. https://doi.org/10.29309/TPMJ/2021.28.08.4721

\section{INTRODUCTION}

It is an indescribable misfortune how an unborn life meets its demise prior to its complete expulsion from the womb it rests in.Intrauterine fetal death (IUFD) has for a long time been an abandoned area of interest. Intrauterine fetal death refers to babies with no signs of life in utero. ${ }^{1}$ Awareness about the factors that led to fetal demise can be helpful both for parents and clinicians to tackle with the situation and to take measures in advance to prevent such unpleasant outcomes. ${ }^{1-3}$

Still birth is defined by the Perinatal Mortality Surveillance Report (CEMACH) "as a baby born with no signs of life known to have died after 24 weeks of gestation." The estimated global number of stillbirths was 2.64 million (uncertainty range, 2.14-3.82 million). ${ }^{4}$ The worldwide stillbirth rate declined from 22.1 stillbirths per 1000 births in 1995 to 18.9 stillbirths per 1000 births in 2009 . Rates of still-births differs from country to country, for instance, according to the Indian census of 2006, the still birth rate in India was 9 per thousand births in rural India and 8 in 1000 births in the urban India. ${ }^{5}$ These contrasting stillbirth rates between rural and urban areas reveal most stillbirths are avoidable and can be prevented with vigilant obstetric care.

It is very hard to understand the root cause underlying fetal demise and there can be multiple factors associated with it. ${ }^{6}$ The depiction is even more complex when there are several underlying causes leading to this worse outcome and it's 
difficult to ascertain one specific etiology. ${ }^{6,7}$ Demographically, age group $>35$ years weighing $>85 \mathrm{~kg}$ go through higher occurrences of fetal demise, while several medical disorders like hypertension, diabetes, thyroid disease, kidney disease, asthma, thrombophilias are also associated with intrauterine deaths. In addition, many deaths remain unexplained.

Diverse classification systems have been employed for diagnosing fetal death, amongst which are the Wigglesworth classification ${ }^{8}$ system and the relevant condition at death (ReCoDe) system. ${ }^{9}$ These categorizing systems can help to delineate the maternal and fetal causes of Intrauterine fetal demise..$^{9,10}$ Based on these systems, the percentage of fetal deaths that remain unexplained can fluctuate from $14.3 \%$ to $47.4 \% .^{11}$

Thus our aims in this study were to analyze different risk factors for stillbirth in the second and third trimesters; early identification of timingspecified risk factors for stillbirth helps physicians to reduce preventable factors and stillbirths, and improves general outcomes of pregnancy.

\section{MATERIAL \& METHODS}

This was a single-center retrospective study conducted at Aga Khan Hospital for women Garden Karachi which is a secondary care facility catering predominantly lower and middle class community. The study was approved by Hospital Ethics Research Committee (4746-Obs-ERC-17).

Retrospective review of medical records of patients over a period of five years from Jan 2012 to Dec 2016 was done. The details were entered in a preformed proforma. The details of complaints at admission, obstetrics history, menstrual history, and examination findings, per vaginal examination findings, mode of delivery and fetal outcomes, placental examination, condition of cord and investigation reports were recorded. Babies born below 24 weeks of gestation, fetuses weighing below $1000 \mathrm{~g}$ at birth, intrapartum stillbirths and twin gestations were excluded. The complaints included a period of amenorrhea, duration of labor pains, history of leaking, bleeding per vaginum (PV), pregnancy induced hypertension $(\mathrm{PIH})$ or eclampsia, decreased or loss of fetal movements. The obstetrical history included parity, abortions, stillbirth, neonatal death, lower segment cesarean section (LSCS), preterm delivery, antepartum hemorrhage (APH) or $\mathrm{PIH}$ in a previous pregnancy. The records of per vaginal findings included bleeding PV, dilatation of the cervix, effacement of the cervix, presenting part, presence of membranes, pelvis, hand prolapse, or cord prolapse. The details of the mode of delivery included vaginal delivery, LSCS, forceps, and laparotomy. Fetal outcomes recorded included fresh/macerated stillbirth, sex of the baby, weight, congenital malformations (CMFs), and birth injuries. Findings of placenta like infarction, calcification, and retroplacental clot and of conditions of the cord like knots, cord around neck, and any other abnormality were also recorded.

The data analysis was carried out employing SPSS 18.0 version. The quantitative variables were reported as mean and standard deviation. Qualitative variables were reported as percentages.

\section{RESULTS}

The study consisted of 56 cases of intrauterine fetal demise out of 4813 number of deliveries which occurred during the study duration. Overall estimated rate of intrauterine fetal demise was $11.6 \%(56 / 4813)$. Out of 56 cases of IUFD, 26 cases (47.3\%) were preterm and 16 cases $(29.1 \%)$ were at term and 13 cases (23.6\%) were post term. Majority of the cases $\mathrm{n}=32$ were multigravida $(58.2 \%)$ and $41.8 \%$ were primigravida $(n=23) .55 .4 . \%$ of the patients were aged less than 30 years with the mean age of 27.58 (Std. deviation $=5.44$ ) and most of them were overweight (59.6\%) with mean BMI of 26.5 and standard deviation of $\pm 4.55 .46 .5 \%(n=26)$ of patients were admitted with the non-specific symptoms, 4 patients presented with absent fetal movements $(7.1 \%)$ while $21.4 \%(n=12)$ came with decreased fetal movements. $14.3 \%(n=8)$ of patients were admitted with abdominal pain and $10.7 \%(n=6)$ with vaginal bleed. $10.7 \%(n=6)$ of cases had Rh -ve status (Table-I). 
(Table-II) Approximately $33.9 \%$ of cases did not have a clear cause of fetal death identified and hence were un-explained. In 56 cases, $28.6 \%$ had some form of congenital abnormality identified on ultrasound, 7.1\% $(n=4)$ had anemia, 23.2\% $(n=13)$ IUGR, 5.4\% $(n=3)$ placental abruption and $1.8 \%$ $(n=1)$ of gestational diabetes.3.9\% $(n=47)$ had normal vaginal delivery and 7 were delivered by LSCS and only 1 patient had instrumental delivery. Fetal characteristics identified $50 \%(n=28)$ were males and $50 \%(n=28)$ were females. 4 fetuses were born with tight cord around their neck $58.1 \%$ were macerated stillbirths $(n=25)$ while $32 \%$ were fresh stillbirth (Table-III).

\begin{tabular}{|l|c|c|}
\hline \multicolumn{1}{|c|}{ Maternal Characteristics } & Number of Cases & Percentage \\
\hline AGE & & 55.4 \\
$<30$ Years & 31 & 44.6 \\
\hline 30 Years & 25 & Mean $=27.58 \pm 5.44$ \\
\hline BMI & & 3.8 \\
Underweight & 2 & 36.5 \\
\hline Normal & 19 & Mean $=26.579 \pm 4.5559 .6$ \\
\hline Overweight & 31 & \\
\hline Booking Status & & 80.4 \\
\hline Booked & 45 & 19.6 \\
\hline Un-booked & 11 & 41.8 \\
\hline Parity & & 58.2 \\
\hline Primiparous & 23 & \\
Multiparous & 32 & 47.3 \\
\hline Gestational Age & & 29.1 \\
Pre-term & 26 & 23.6 \\
\hline Term & 16 & 10.7 \\
\hline Post-term & 13 & \\
\hline Rh-ve Status & 6 & 7.1 \\
\hline Admitting Complaints & & 21.4 \\
Absent fetal movements & 4 & 14.3 \\
Decreased fetal movements & 12 & 10.7 \\
\hline Abdominal pain & 8 & 46.5 \\
\hline Vaginal bleed/leak & 6 & \\
Non-specific symptoms & 26 & \\
\hline & Table-I. Maternal characteristics (n=56). & \\
\hline
\end{tabular}

\begin{tabular}{|l|c|c|}
\hline \multicolumn{1}{|c|}{ Maternal Characteristics } & Number of Cases & Percentage \% \\
\hline Unexplained Causes & 19 & 33.9 \\
\hline Congenital anomaly & 16 & 28.6 \\
\hline Intrauterine growth retardation & 13 & 23.2 \\
\hline Placental Abruption/Gestational Hypertension & 3 & 5.4 \\
\hline Gestational Diabetes & 1 & 1.8 \\
\hline Anemia & 4 & 7.1 \\
\hline
\end{tabular}

Table-II. Causes of intrauterine fetal demise $(n=56)$.

\begin{tabular}{|l|c|c|}
\hline \multicolumn{1}{|c|}{ Fetal Characteristics } & Number of Cases & Percentage \% \\
\hline Sex female & 28 & 50 \\
Male & & 50 \\
female & 28 & 58.1 \\
\hline Macerated still birth & 25 & 32.6 \\
\hline Fresh stillbirths & 14 & 9.3 \\
\hline Tight cord around neck & 4 & \\
\hline
\end{tabular}




\section{DISCUSSION}

According to Every Newborn Action Plan (ENAP) 2014, it was decided in World Health Assembly that stillbirth rate shall be $12 / 1000$ worldwide and majority of high income countries achieved this target by 2015 although the timeline was 2030 . Still 2.6 million stillbirths were recorded in 2015, $98 \%$ being in low and middle income countries. Two thirds of stillbirths happen in ten countries and Pakistan is third in the list ranking for total stillbirths (243000) and top most in the world having a stillbirth rate $43.1 / 1000$ births. ${ }^{12}$

Our study targeted at antepartum stillbirths, weighing more than 1000 grams and gestational age more than 24 weeks, though lancet series of still births took 28 weeks of gestation as cut off keeping in view the neonatal intensive care facilities in developing countries. A fetal death rate (stillbirth) of $56 / 4813(11.6 \%)$ is computed from our analysis. A similar study from India reported $16 / 1000$ births, as they took into account both intrapartum and antepartum stillbirths. ${ }^{13}$ Another study reported $36 / 1000$ births ${ }^{15}$ A study from Taiwan over a period of ten years reported $0.98 \%$ incidence of intrauterine fetal demise ${ }^{14}$ and reported incidence from an African study over a period of one month was $8 \% .{ }^{16}$ These different rates and incidences are attributable to varying study durations, geographical locations and study designs.

Our hospital being a secondary care centre located at a site catering lower and middle class population is likely to have a raised intrauterine fetal death rate on account of socio demographic location and well recognized risk factors; illiteracy and poor socioeconomic status. These have been identified in majority of studies as contributing factors for antepartum stillbirths. ${ }^{13,14,15,16}$ Majority of the data reported in literature is from tertiary care hospitals which serve as a referral centre for all high risk cases thus reporting higher rates of still births, still we need to get information on all those still births too that never come in contact with any health care professional and are never recorded, thus emphasizing the need for vital statistics.
In our study majority of women were preterm (47.3\%), $29.1 \%$ at term and $23.6 \%$ were post term. Similar results were reported in another study, $55.4 \%$ in second trimester and $44.6 \%$ in third trimester with only $14.9 \%$ at term. ${ }^{14}$ Prematurity is an important contributor to perinatal morbidity and mortality and has been identified as an important contributor for intrauterine deaths and still births in various studies like ours. ${ }^{17}$

Congenital anomalies were responsible for $28.6 \%$ of stillbirths In the case of stillbirths with congenital malformations or chromosomal abnormalities, it's important to counsel parents regarding importance of nuchal translucency testing in first trimester and further invasive prenatal testing if required. Another study reported $19 \%$ of stillbirths due to congenital anomalies and $46 \%$ unexplained in antepartum period though placental insufficiency and hypertensive disorders contributing to $20 \%$ of stillbirths however diabetes contributing only $1.6 \%$ similar to ours. ${ }^{13}$ Similar results reporting hypertensive disorders as most common medical disorder responsible were reported in another study and unexplained attributing to $30 \% .{ }^{14}$ In those pregnancies which are considered high risk; careful antenatal surveillance is paramount to avoid the chunk of unexplained causes of stillbirth.

Our data revealed that $23.2 \%$ of stillbirths were associated with Small for gestational age (SGA) fetuses which are defined as birth weight below the 10th percentile for gestational age. The fetal weight is particularly smaller in Asia than in America. ${ }^{15}$ Serial Ultrasound scans with strict antenatal surveillance using population based growth charts followed by timely delivery are paramount in order to prevent fetal demise associated with SGA fetuses.

Lancet series attributes majority of stillbirths to maternal infections like malaria, syphilis and others to non-communicable diseases and very few to anomalies. ${ }^{12}$ We have checked medical records for any history of infections but the study being retrospective no infection screens could be done and records did not show many cases of infection. This may be due to the fact that economic 
issues come in after a still birth and families usually are unable to finance investigations on account of their social status. The umbrella of contributing factors varies with the ethnicity and the prevalence of medical conditions in the areas studied.

In our study $58.2 \%$ were multigravidas and rest primigravidas. A study reported more cases in multigravidas. ${ }^{18}$ Though primigravidas are considered more vulnerable ${ }^{16}$, nearly equal contribution was seen from primi and multigravidas in another study..$^{17}$ Age more than 35 years and $\mathrm{BMI}>30$ are also important contributors as in our study we took age > 30 years and had $44.6 \%$ in this group though age $<30$ years contributed more to stillbirths in our study group. This can be attributed to the sociocultural context of Pakistan with earlier marriages and majority has completed families by the age of 35 . Similar results are reported from Indian studies being the same cultural and geographical areas. ${ }^{13,15,18}$ Increased BMI has also been reported as a significant contributor in Lancet series and study from Taiwan where they have looked at stillbirths in trimesters and smoking habits and BMI contributing most towards third trimester. ${ }^{12,14}$ We did check our records for smoking status but did not find significant details. Although being a tertiary care hospital and receiving referrals and non-booked cases, our booked cases having intrauterine fetal demise were around $80 \%$ which is in contrast to other studies reported where non booked contribute more. ${ }^{16,18}$ In our case the reason might be poor antenatal care, poor compliance by the women to the treatments offered, congenital anomalies, BMI, past history of still birth and most importantly poor health seeking behaviors.

Complaints at the time of admission were in the majority of cases (46.5\%) not significant with only $21.4 \%$ admitted with decreased fetal movements and $7.1 \%$ with absent fetal movements. Few studies have looked at the presenting complaints at the time of admission as majority focus on intrapartum events like color of liquor, amount of bleeding. ${ }^{13}$ There were $50 \%$ males and $50 \%$ females which is similar to literature showing no association of gender with the stillbirths though some studies reporting males being the dominant gender in stillbirths. ${ }^{14}$

In view of literature reported our study is an attempt to add to the previous analysis of causes and determinants of intrauterine fetal deaths published from Pakistan. This study has almost comparable results in relation to maternal age, BMI, causes of intrauterine fetal deaths, macerated still births at the time of birth and vaginal deliveries, to the literature previously published from Pakistan except for the booking status of patients. ${ }^{19-22}$

This study aimed to look only at antepartum intrauterine fetal deaths reporting to our center in the last 5 years and identifying the factors mainly responsible for antepartum period cases. The strength of the study was that it looked only one specific time period and had sufficient cases from last 5 years and very comprehensive information as detailed patient records were available. Limitations being retrospective and no investigations could be planned or any further information desired could not be obtained.

This study looked into the impact of factors like improved antenatal care and health education, that previous literature have identified as major determinants for reducing stillbirths and it was observed that although majority of patients were booked cases, still births were high. Besides emphasizing on antenatal care and patient education and compliance, two other important aspects need to be considered that is to see whether the quality of care being provided at the health care centers is by trained health care professionals who are up to date with latest advancements and research in the field to provide their clients the highest standards of care and treatments. Secondly the patients coming for antenatal visits are compliant with the treatments and nutrition advise and there is a concept of universal coverage or health insurance, ensuring them to finance themselves for hospital based care, delivery and treatments.

\section{LIMITATIONS}

Our study being retrospective and single centre 
based like many other studies from Pakistan, suggest the need to do prospective cohort at national level in all teaching and secondary care centers to have national data base. This will help us looking at exact figures and to measure associations between various sociodemographic and medical factors responsible. This might lead policy makers to have household survey and national statistics thus having strategies planned for the ENAP targets to be achieved by 2030 .

\section{CONCLUSION}

Antepartum intrauterine fetal death or stillbirths in the third trimester, affect the entire family, both parents and the other siblings who are looking forward to the upcoming bundle of joy. Modifiable factors identified in the preconception and antenatal period need access to quality health care services and provision of universal health coverage for all. We conclude from our study that quality antenatal care with health education and timely interventions can reduce the number of intrauterine fetal deaths thus reducing the psychological sequelae.

Copyright@ 30 Nov, 2020.

\section{REFERENCES}

1. Late intrauterine fetal death and stillbirth. RCOG Green top guideline no. 55 .

2. Faye-Petersen OM, Guinn DA, Wenstrom KD. Value of perinatal autopsy. Obstet Gynecol 1999; 94:915-920.

3. Michalski ST, Porter J, Pauli RM. Costs and consequences of comprehensive stillbirth assessment. Am J Obstet Gynecol 2002; 186:10271034.

4. Hickey L, Murphy A, Devaney D, Gillan J, Clarke T. The value of neonatal autopsy. Neonatology 2012; 101 :6873.

5. Estimates of mortality indicators-Census of India website-vital statistics. Chapter $4, \mathrm{Pg} 81$, statement 51, 2010.

6. Censusindia. Available at. Censusindia.gov.in/vital statistics/srs/Chap_4_- 2010.pdf. Accessed on $2 \overline{5}$ February 2016.

7. Silver RM. Fetal death. Obstet Gynecol 2007; 109:153167.
8. Silver RM, Varner MW, Reddy $U$, et al. Work-up of stillbirth: A review of the evidence. Am J Obstet Gynecol 2007; 196: 433-444.

9. Wigglesworth JS. Monitoring perinatal mortality. A pathophysiological approach. Lancet 1980; 2:684-686.

10. Gardosi J, Kady SM, McGeown P, Francis A, Tonks A. Classification at death (ReCoDe): population based cohort study. BMJ 2005; 331:1113-1117.

11. Reddy UM, Goldenberg R, Silver R, et al. Stillbirth classification- developing an international consensus for research: Executive summary of a National Institute of Child Health and Human Development Workshop. Obstet Gynecol 2009; 114:901-914.

12. Vergani $\mathrm{P}$, Cozzolino S, Pozzi E, et al. Identifying the causes of stillbirth: a comparison of four classification systems. Am J Obstet Gynecol 2008; 199:319.e1-319.e4.

13. Lawn JE et al. Ending preventable stillbirths 2. Stillbirths: rates, risk factors, and acceleration towards 2030. The Lancet 2016; 387:587-603. http://dx.doi. org/10.1016

14. Newtonraj A, Kaur M, Gupta M, Kumar R. Level, causes, and risk factors of stillbirth: A population-based case control study from Chandigarh, India. BMC Pregnancy and Childbirth 2017; 17:371 DOI 10.1186/ s12884-017-1557-4.

15. Liu LC, Huang HB, Yu MH, Su HY. Analysis of intrauterine fetal demise- A hospital based study in Taiwan over a decade. Taiwanese Journal of Obstetrics and Gynecology 2013; 52: 546-550.

16. W.J. Kierans, K.S. Joseph, Z.C. Luo, R. Platt, R. Wilkins, M.S. Kramer Does one size fit all? The case for ethnic-specific standards of fetal growth. BMC Pregnancy Childbirth, 8 (2008), p. 1.

17. Sharma S, Sidhu H, Kaur S. Analytical study of intrauterine fetal death cases and associated maternal conditions. Int J Appl Basic Med Res. 2016; 6(1): 11-13. doi: 10.4103/2229-516X.173986.

18. Tilahun $D$, Assefa $T$. Incidence and determinants of stillbirth among women who gave birth in Jimma University specialized hospital, Ethiopia. The Pan African Medical Journal. 2017; 28:299. doi:10.11604/ pamj.2017.28.299.1269.

19. Garg S, Kumar N. Analysis of factors responsible for intrauterine fetal death in rural pregnant women at tertiary care centre of Northern India. Int J Reprod Contracept Obstet Gynecol. 2017; 6(9):4071-4074. DOI: http://dx.doi.org/10.18203/2320-1770.ijrcog20174064 
20. Munim S, Nawaz FH, Ayub S. Still births - eight years' experience at Aga Khan University Hospital Karachi, Pakistan. The Journal of Maternal-fetal And Neonatal Medicine 2011; 24(3): 449-452. https://doi.org/10.3109 /14767058.2010.482619.

21. Malik R, Khan NR, Arif A, Ayoub S, Perveen S. Common risk factors in patients with intrauterine fetal death. $J$ Postgrad Med Inst 2016; 30(4): 337-41.
22. Perveen F, Tayyab S, Zuberi BF. Risk factors for perinatal deaths in Pakistan. The Journal of Obstetrics and Gynecology Research 2011; 37(10): 1359-1364. https://doi.org/10.1111/j.1447-0756.2011.01536.x.

23. Jehan I et al. Stillbirths in an urban community in Pakistan. Am J Obstetr Gynecol 2007; 197(3): 257e1257e8. https://dx.doi.org/10.1016\%2Fj.ajog.2007.07. 012.

\begin{tabular}{|l|l|l|}
\hline \multicolumn{3}{|c}{ AUTHORSHIP AND CONTRIBUTION DECLARATION } \\
\hline Sr. \# & \multicolumn{1}{|c|}{ Author(s) Full Name } & Contribution to the paper \\
\hline 1 & Erum Saleem Khan & Principal Investigator. \\
\hline 2 & Mahwash Jamil & Manuscript writer. \\
\hline 3 & Reeta Chanderparkash & Data collection and analysis. \\
\hline 4 & Sheikh Irfan & Research associate. \\
\hline 5 & Sana Yousf & Research associate. \\
\hline 6 & Ayesha Basharat & Manuscript writer. \\
\hline
\end{tabular}

